\section{A portable high-intensity focused ultrasound system for the pancreas with 3D electronic steering: a preclinical study in a swine model}

\author{
Won Chang', Jae Young Lee 2 , Jae Hwan Lee ${ }^{3}$ Jae Seok Bae ${ }^{4}$, Yeon Jin Cho ${ }^{4}$, \\ Kook Jin Kang ${ }^{5}$, Keonho Son ${ }^{5}$, Yul Ri Chung ${ }^{6}$, Kyoung Bun Lee ${ }^{7}$, Joon Koo Han ${ }^{2}$ \\ 'Department of Radiology, Seoul National University Bundang Hospital, Seongnam; \\ ${ }^{2}$ Department of Radiology and the Institute of Radiation Medicine, Seoul National University \\ Hospital, Seoul; ${ }^{3}$ Department of Radiology, National Cancer Center, Goyang; ${ }^{4}$ Department \\ of Radiology, Seoul National University Hospital, Seoul; ${ }^{5}$ Therapeutic Ultrasound Division, \\ Alpinion Medical Systems Co., Ltd., Seoul; ${ }^{6}$ Department of Pathology, Seoul National \\ University Bundang Hospital, Seongnam; ${ }^{7}$ Department of Pathology, Seoul National \\ University Hospital, Seoul, Korea
}

Purpose: The aim of this animal study was to evaluate the safety and feasibility of a portable, ultrasonography-guided, high-intensity focused ultrasound (USg-HIFU) system to treat the pancreas.

Methods: Eight swine were included. Using a portable HIFU device (ALPIUS 900, Alpinion Medical Systems), ablations were performed on the pancreas in vivo. Different acoustic intensities were applied $\left(1.7 \mathrm{~kW} / \mathrm{cm}^{2}\right.$ or $1.5 \mathrm{~kW} / \mathrm{cm}^{2}, \mathrm{n}=2$ [group A for a pilot study]; $1.5 \mathrm{~kW} /$ $\mathrm{cm}^{2}, \mathrm{n}=3$ [group B]; and $1.2 \mathrm{~kW} / \mathrm{cm}^{2}, \mathrm{n}=3$ [group C]). Magnetic resonance imaging (MRI) was performed immediately (group A) or 7 days (groups B and C) after HIFU treatment. In groups B and $C$, serum amylase and lipase levels were measured on days 0 and 7 , and performance status was observed every day. Necropsy was performed on days 0 (group A) or 7 (groups B and C) to assess the presence of unintended injuries and to obtain pancreatic and peripancreatic tissue for histological analysis.

Results: Ablation was noted in the pancreas in all swine on MRI, and all pathologic specimens showed coagulation necrosis in the treated area. The mean ablation areas on MRI were $85.3 \pm 38.1 \mathrm{~mm}^{2}, 90.7 \pm 21.2 \mathrm{~mm}^{2}$, and $54.4 \pm 30.6 \mathrm{~mm}^{2}$ in groups $A, B$, and $C$, respectively $(P>0.05)$. No animals showed evidence of complications, except for one case of a pseudocyst in group $B$.

Conclusion: This study showed that pancreas ablation using a portable USg-HIFU system may be safe and feasible, and that coagulation necrosis of the pancreas was successfully achieved with a range of acoustic intensities.

Keywords: High-intensity focused ultrasound; Animal research; Pancreas

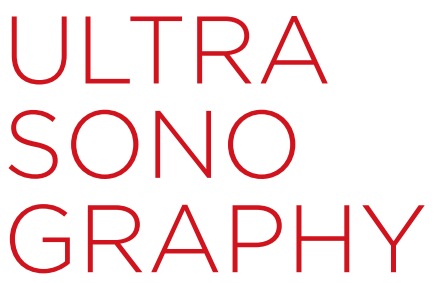

\section{ORIGINAL ARTICLE}

https://doi.org/10.14366/usg. 17048 pISSN: 2288-5919 e elSSN: 2288-5943 Ultrasonography 2018;37:298-306

Received: June 29, 2017

Revised: October 11, 2017

Accepted: October 13, 2017

Correspondence to: Jae Young Lee, MD, Department of Radiology and the Institute of Radiation Medicine, Seoul National University Hospital, 101 Daehak-ro, Jongno-gu, Seoul 03080, Korea

Tel. +82-2-2072-3073

Fax. +82-2-743-6385

E-mail: leejy4u@snu.ac.kr

This is an Open Access article distributed under the terms of the Creative Commons Attribution NonCommercial License (http://creativecommons.org/ licenses/by-nc/3.0/) which permits unrestricted noncommercial use, distribution, and reproduction in any medium, provided the original work is properly cited.

Copyright (C) 2018 Korean Society of Ultrasound in Medicine (KSUM)

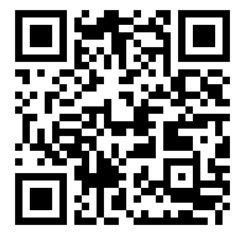

How to cite this article:

Chang W, Lee JY, Lee JH, Bae JS, Cho YJ, Kang KJ, et al. A portable high-intensity focused ultrasound system for the pancreas with 3D electronic steering: a preclinical study in a swine model. Ultrasonography. 2018 0ct;37(4):298306. 


\section{Introduction}

Pancreatic cancer is the twelfth most common cancer and the seventh leading cause of cancer-related deaths in the world [1]. Although surgery is a potentially curative option for pancreatic cancer, only $15 \%-20 \%$ of patients are suitable for surgical resection due to the late presentation of the disease [2]. Therefore, many patients with advanced-stage pancreatic cancer undergo alternative treatments, such as systemic chemotherapy or concurrent chemoradiotherapy $[3,4]$. Although the survival rates have improved over the decades, the 5-year survival rate is still less than $8 \%$ [5]. Therefore, attempts should be made to enhance treatment outcomes in pancreatic cancer patients who cannot be treated surgically.

High-intensity focused ultrasound (HIFU) is a minimally invasive technology in which high-intensity acoustic energy from an extracorporeal source can be focused in the body without introducing a device into the body. HIFU ablation has been used to treat a wide variety of both benign and malignant tumors that are accessible by ultrasound (US) energy [6]. Moreover, HIFU offers an additional treatment opportunity for patients without any other available options [7].

HIFU ablation is performed under imaging guidance. According to the guidance modality, HIFU machines can be divided into magnetic resonance-guided HIFU (MRg-HIFU) and ultrasonography-guided HIFU (USg-HIFU). MRg-HIFU allows the temperature of the targeted tissue to be monitored, which is helpful for ensuring targeting safety and monitoring the treatment response during HIFU treatment. This function is considered one of the advantages of MRg-HIFU compared to USg-HIFU. However, USg-HIFU can provide real-time imaging during treatment, which is critical for the treatment of moving organs such as the pancreas.

A new portable USg-HIFU unit (ALPIUS 900, Alpinion Medical Systems, Seoul, Korea) was recently introduced; this unit provides a targeted forecasting function that displays where the US beams are focused by insonating short pulses before HIFU treatment. This function helps enhance the targeting safety and treatment monitoring to some extent, similar to temperature monitoring in MRg-HIFU [8-10]. Real-time imaging with this USg-HIFU unit involves high imaging frame rates, and the interleaved image display helps ensure that the targeting focus is within the boundary of moving intra-abdominal organs such as the pancreas, liver, and kidneys. In addition, this device has a flexible positioning arm and a small treatment head, which could provide much more spatial flexibility in treatment planning, especially for intra-abdominal organs. Most commercialized HIFU devices are large and immobile, which hinders their use in a small space and prevents HIFU from being applied broadly $[11,12]$. However, this novel device has small dimensions (1.8 m long, $1.2 \mathrm{~m}$ wide, and $1.6 \mathrm{~m}$ high), and the presence of four wheels under the main body enables it to be moved. A previous study showed that this system was safe and accurate for the ablation of fixed soft tissue, but it was not evaluated for mobile organs such as the pancreas [13].

Previous preclinical studies have shown that USg-HIFU is safe and feasible for use in treating pancreatic lesions [14,15], and early clinical studies have demonstrated that HIFU alone could palliate cancer-related pain in patients with unresectable pancreatic cancer $[16,17]$. Furthermore, the combined use of chemotherapy and HIFU may be beneficial $[7,18]$.

Therefore, the aim of this study was to evaluate the safety and feasibility of this new portable USg-HIFU system for treating the pancreas in a swine model.

\section{Materials and Methods}

\section{Animals}

This study was approved by our Institutional Animal Care and Use Committee. All experiments were conducted in accordance with the institutional guidelines. Eight male domestic pigs weighing 50-60 $\mathrm{kg}$ were used in our study.

The pigs were not fed for 12 hours before HIFU treatment to decrease the extent to which bowel gas limited the visualization of the pancreas on US. Each animal was sedated with an intramuscular injection of zolazepam ( $5 \mathrm{mg} / \mathrm{kg}$, Zoletil, Virbac, Carroscedex, France) and xylazine (10 mg/kg, Rompun, Bayer-Schering Pharma, Berlin, Germany), and the animals were then intubated and ventilated during the procedures. Anesthesia was maintained by the inhalation of $1 \%-3 \%$ isoflurane in pure oxygen gas. To facilitate US propagation through the skin, the pigs underwent shaving and subsequent waxing of their abdomens just before treatment. During the entire HIFU procedure, each animal's vital signs, including the pulse rate, electrocardiogram, and temperature, were carefully monitored.

\section{HIFU Treatment}

The new portable USg-HIFU system (ALPIUS 900, Alpinion Medical Systems) was used in our study (Fig. 1). It has a flexible positioning arm and a treatment head that is usually incorporated into HIFU devices for thyroid and breast lesions $[19,20]$. The treatment head contains both a HIFU treatment transducer and an imaging transducer. It is easily adjusted by hand, provides sufficient spatial flexibility in treatment planning, and creates better sonic windows into the pancreas. This system has a phased-array 256-channel transducer (radius of curvature, $9 \mathrm{~cm}$; aperture size, $15 \mathrm{~cm}$ ) that can perform volumetric ablation without repositioning the transducer, 


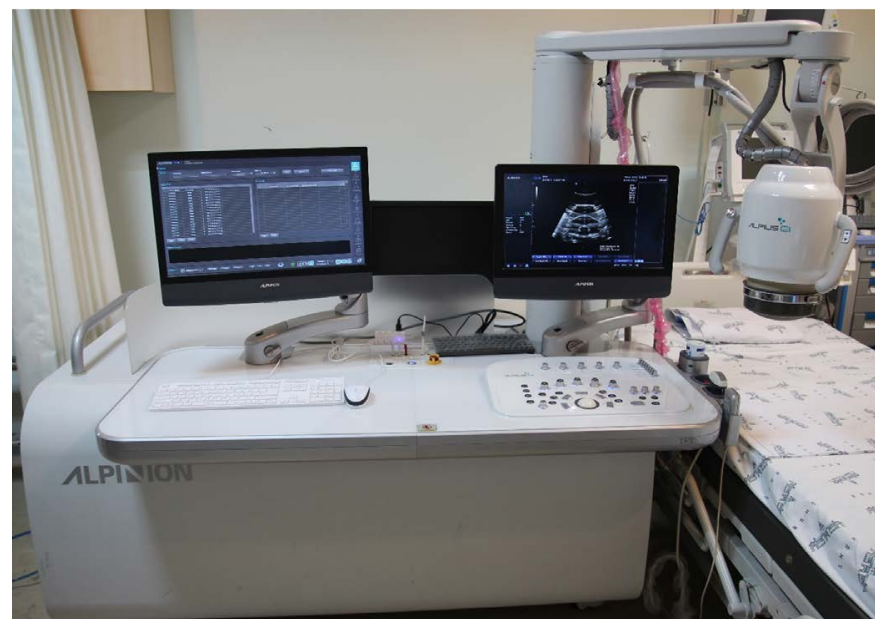

A

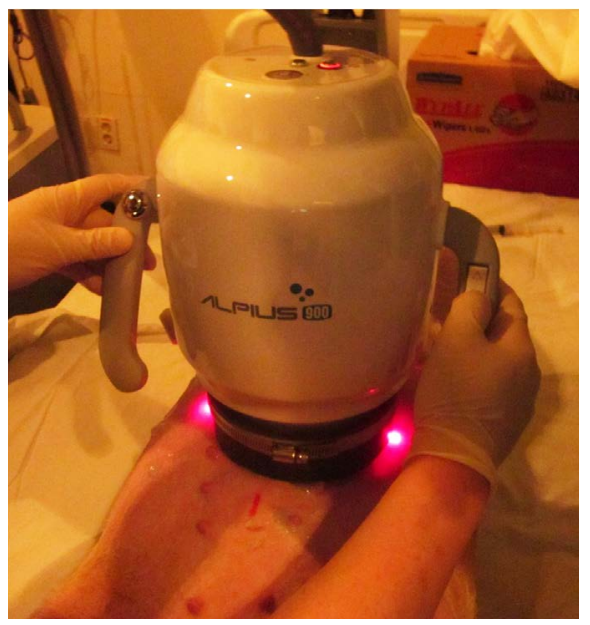

B

Fig. 1. Photographs of the portable ultrasonography-guided high intensity focused ultrasound (HIFU) system.

A. An overview of the HIFU system (ALPIUS 900) used in this study is shown. B. The treatment head is easily adjusted by hand.

Table 1. Treatment parameters

\begin{tabular}{|c|c|c|c|c|c|c|c|c|c|c|c|c|}
\hline Group & Pig & $\begin{array}{c}\text { Acoustic } \\
\text { intensity in situ } \\
\left(\mathrm{kW} / \mathrm{cm}^{2}\right)\end{array}$ & $\begin{array}{c}\text { Acoustic } \\
\text { power (W) }\end{array}$ & $\begin{array}{c}\text { Exposure } \\
\text { duration per } \\
\text { point (sec) }\end{array}$ & $\begin{array}{c}\text { No. of } \\
\text { ablation } \\
\text { points }\end{array}$ & $\begin{array}{c}\text { Inter-point } \\
\text { movement } \\
\text { time (sec) }\end{array}$ & $\begin{array}{c}\text { Duty cycle } \\
(\%)\end{array}$ & $\begin{array}{c}\text { Pulse repetition } \\
\text { frequency }(\mathrm{Hz})\end{array}$ & $\begin{array}{l}\text { AWT } \\
(\mathrm{mm})\end{array}$ & $\begin{array}{l}\text { DSF } \\
(\mathrm{mm})\end{array}$ & $\begin{array}{l}\text { DPF } \\
(\mathrm{mm})\end{array}$ & $\begin{array}{c}\text { Acoustic } \\
\text { energy in } \\
\text { situ (J) }\end{array}$ \\
\hline \multirow[t]{2}{*}{ A } & 1 & 1.7 & 320 & 8 & 9 & 2 & 80 & 10 & 27.8 & 64.2 & 80.5 & 2,048 \\
\hline & 2 & 1.5 & 285 & 7 & 9 & 2 & 80 & 10 & 25.3 & 43.8 & 64.9 & 1,596 \\
\hline \multirow[t]{3}{*}{ B } & 3 & 1.5 & 238 & 7 & 9 & 2 & 80 & 10 & 23.3 & 56.8 & 75.1 & 1,332 \\
\hline & 4 & 1.5 & 199 & 7 & 9 & 2 & 80 & 10 & 21.3 & 44.3 & 70 & 1,114 \\
\hline & 5 & 1.5 & 277 & 7 & 9 & 2 & 80 & 10 & 21.9 & 51.7 & 65.1 & 1,552 \\
\hline \multirow[t]{3}{*}{$C$} & 6 & 1.2 & 155 & 7 & 9 & 2 & 80 & 10 & 17.3 & 37.8 & 65.1 & 866 \\
\hline & 7 & 1.2 & 137 & 7 & 9 & 2 & 80 & 10 & 22.7 & 31.5 & 66 & 769 \\
\hline & 8 & 1.2 & 176 & 7 & 9 & 2 & 80 & 10 & 24 & 40.2 & 65.3 & 898 \\
\hline
\end{tabular}

AWT, abdominal wall thickness; DSF, distance between the skin surface and focus; DPF, distance between the probe surface and focus.

and it has a steering range of $40 \mathrm{~mm}$ along the direction of US propagation and $24 \mathrm{~mm}$ along the direction perpendicular to the US propagation through the electronic steering of the focal spots. The focal size of the transducer has a width of $1.5 \mathrm{~mm}$ and an axial length of $8 \mathrm{~mm}$, with a center frequency of $1 \mathrm{MHz}$ at $-6 \mathrm{~dB}$. During ablation, the transducer can be cooled by the water circulation module placed anterior to the transducer using degassed water at $9^{\circ} \mathrm{C}-15^{\circ} \mathrm{C}$.

For treatment planning and monitoring, a 3.5-MHz ultrasonography transducer is located at the center of the HIFU transducer. The image transducer can rotate within the HIFU transducer without repositioning and provide 3-dimensional (3D) volumetric data for ablation planning.

The splenic lobe of the pancreas was targeted for HIFU in all pigs. After confirming that the HIFU beams were focused properly within the pancreas before HIFU insonation was started, using the targeted forecasting function described above, 9 points were allocated, with 1 point placed at the center and 8 points placed $3 \mathrm{~mm}$ away from the center. Ablation was then started with electronic steering. During HIFU insonation, whether hyperechoic areas were produced within the pancreas was verified on real-time US imaging (Fig. 2).

The first two pigs were used in a preliminary study to check the feasibility of HIFU treatment for the pancreas. They were allocated to group A. The first and second pigs were treated with an acoustic intensity of $1.7 \mathrm{~kW} / \mathrm{cm}^{2}$ and $1.5 \mathrm{~kW} / \mathrm{cm}^{2}$, respectively. The remaining six pigs were randomly allocated into group $B$ or group $C$ and treated using an acoustic intensity of $1.5 \mathrm{~kW} / \mathrm{cm}^{2}$ or $1.2 \mathrm{~kW} / \mathrm{cm}^{2}$, respectively. The other HIFU parameters that were used are shown in Table 1. HIFU intensities lower than $3 \mathrm{~kW} / \mathrm{cm}^{2}$ were used in previous studies without general anesthesia or hospitalization $[17,18,21]$, 
and in a previous clinical study using acoustic intensities of 1-2 $\mathrm{kW} / \mathrm{cm}^{2}$ for the treatment of pancreatic cancer, there were no major complications related to the HIFU treatment [18]. Therefore, three acoustic intensities $\left(1.2 \mathrm{~kW} / \mathrm{cm}^{2}, 1.5 \mathrm{~kW} / \mathrm{cm}^{2}\right.$, and $\left.1.7 \mathrm{~kW} / \mathrm{cm}^{2}\right)$ between $1 \mathrm{~kW} / \mathrm{cm}^{2}$ and $2 \mathrm{~kW} / \mathrm{cm}^{2}$ were chosen for this study to evaluate the safety and feasibility of HIFU treatment using this new machine, in a setting similar to that used in a previous clinical study.

\section{Follow-up and Analysis}

The presence of skin burns and abdominal muscle damage was evaluated immediately after the HIFU procedure. Status observations for activities, dietary intake, and the presence of vomiting, diarrhea, or irritability were conducted daily for all animals in groups B and $C$ as indirect indicators of the presence of unintentional injuries. Blood was sampled prior to and 7 days after the HIFU procedure in groups B and C. Serum amylase and lipase levels were checked as

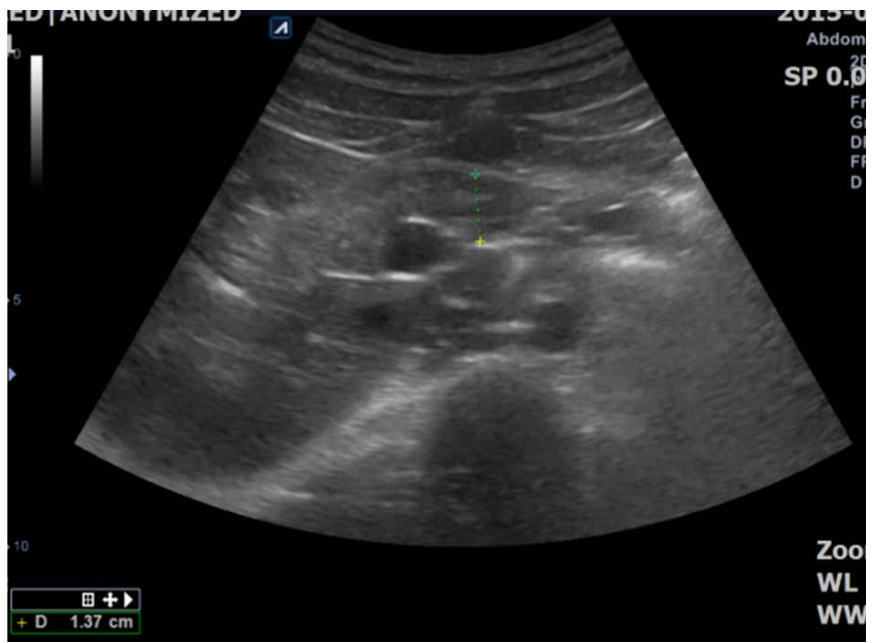

A

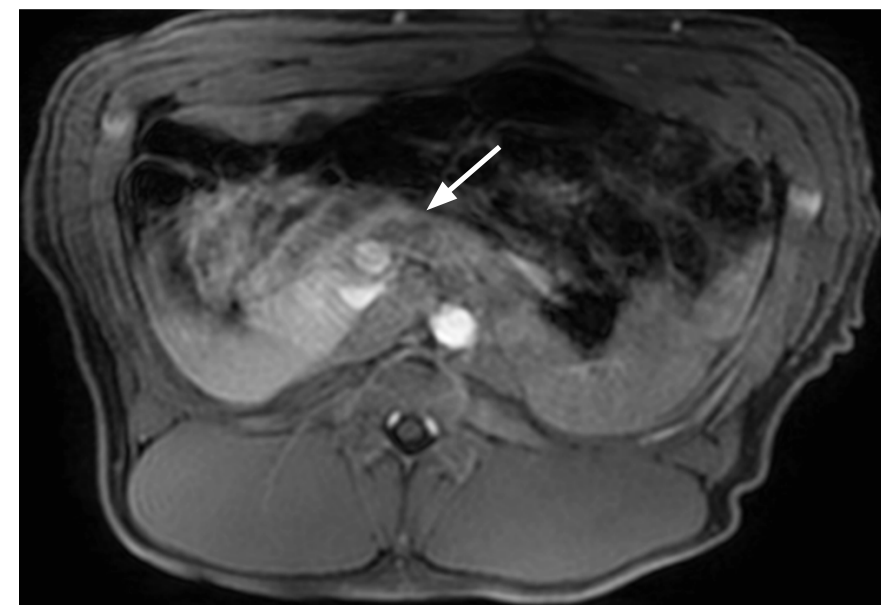

C

Fig. 2. An ablated area on ultrasonography and magnetic resonance imaging (MRI).

A. Ultrasonography shows a swine pancreas with $1.37 \mathrm{~cm}$ thickness. B. Ultrasonography shows a hyperechoic change in the targeted area (arrow). C. T1-weighted MRI of the portal phase shows a poorly enhanced focal lesion in the targeted area (arrow).

biochemical evidence of pancreatitis.

To evaluate the targeting accuracy, ablation size, and the presence of any unintentional injuries before sacrifice, magnetic resonance imaging (MRI) was performed immediately after HIFU treatment in group $\mathrm{A}$ or 7 days after HIFU treatment in groups $\mathrm{B}$ and $\mathrm{C}$ using an Ingenia 3.0-T MRI unit (Phillips Healthcare, Best, the Netherlands) and a body surface coil. T2-weighted fat-suppressed images (slice thickness of $3 \mathrm{~mm}$ with a 3-mm gap) and T1-weighted, fat-suppressed, dynamic contrast-enhanced images (slice thickness of $3 \mathrm{~mm}$ with a 3-mm gap) in the unenhanced arterial phase (15-second delay) and the portal venous phase (90-second delay) were acquired on axial planes. Gadopentetate dimeglumine (Magnevist, Bayer Healthcare) at 0.1 $\mathrm{mmol} / \mathrm{kg}$ was injected intravenously, followed by a saline flush.

To determine the targeting accuracy of the HIFU treatment, the splenic lobe of the pancreas was investigated to evaluate the presence of a poorly enhanced focal lesion in the portal phase,

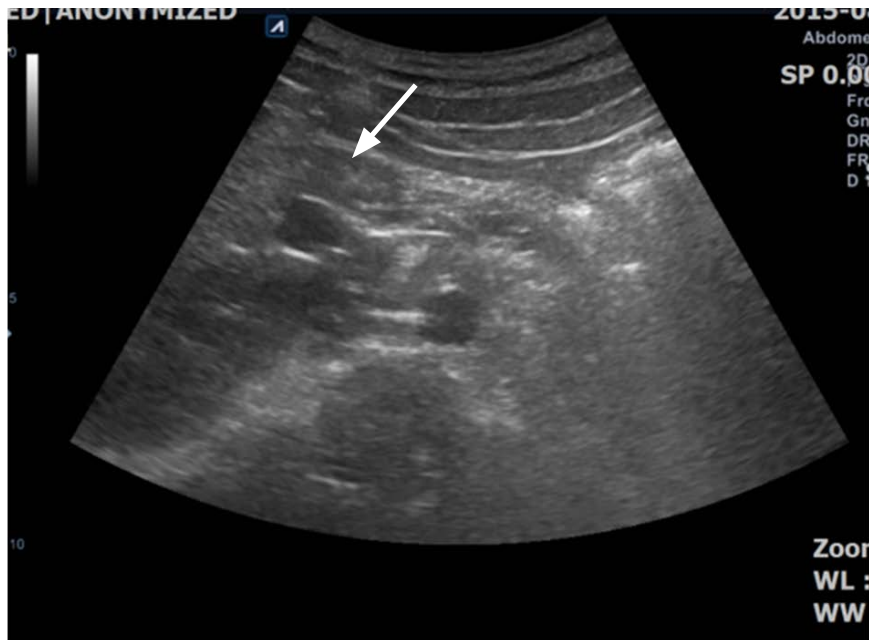

B 
which represented coagulation necrosis. Ablation size was calculated under the assumption of an ellipse. The presence of injury to the abdominal organs, the abdominal wall, and vertebral bodies was also evaluated.

Before the MRI study, the pigs were given $15 \mathrm{mg} / \mathrm{kg}$ of zolazepam hydrochloride (Zoletil, Virbac, Carros, France) and $5 \mathrm{mg} / \mathrm{kg}$ of xylazine (Rumpun, Bayer Korea, Ansan, Korea) intramuscularly for deep sedative anesthesia. Additional anesthetic agents were administered intravenously as needed.

All animals were euthanized just after the MRI study. During necropsy, a thorough gross examination was performed, and the presence of unintended injuries at the skin, abdominal wall, mesentery, or bowel walls was recorded. The pancreas was then resected. The pancreas was fixed in $40 \mathrm{~g} / \mathrm{L}$ of formaldehyde solution, embedded in paraffin, and stained with hematoxylin and eosin for light microscopy.

\section{Statistics}

Due to the small sample, nonparametric statistics were used. For the ablation size, the Kruskal-Wallis $\mathrm{H}$ test was used, and for paired data such as amylase and lipase levels, the Wilcoxon signed-rank test was used. P-values $<0.05$ were considered to indicate significant differences.

\section{Results}

\section{Treatment Monitoring, Gross Examination, Status Observations, and Biochemical Analysis}

An adequate sonic window for the splenic lobe was obtained, and HIFU treatment was successfully administered to all pigs. The mean thickness of the pancreas was $1.16 \mathrm{~cm}$ (range, 0.92 to $1.53 \mathrm{~cm}$ ). All pigs tolerated the HIFU treatment, and their vital signs were stable during the procedure. After treatment, no skin burns or abdominal wall injuries were observed on gross examination.

No significant abnormalities were observed on daily status observations in groups $B$ and $C$. The mean serum lipase levels on day 0 and day 7 were $7.7 \pm 2.3 \mathrm{U} / \mathrm{L}$ and $6.8 \pm 2.3 \mathrm{U} / \mathrm{L}$, respectively, and the mean serum amylase levels on day 0 and day 7 were $29.9 \pm 9.3$ $\mathrm{U} / \mathrm{L}$ and $31.7 \pm 6.8 \mathrm{U} / \mathrm{L}$, respectively. No significant interval changes were observed in the serum lipase and amylase levels between days 0 and 7 ( $P>0.05$, both).

\section{MRI Findings}

Focal areas of poor enhancement on the portal phase of T1weighted images, representing coagulation necrosis, were created exclusively within the pancreas in all pigs, except for one case. In one pig (pig 3) in group B, a pseudocyst (18.7 $\mathrm{mm} \times 17.7 \mathrm{~mm}$ on the axial plane) developed within the pancreas without evidence of adjacent organ injury. Excluding the case with pseudocyst formation, the mean ablation areas were $85.3 \pm 38.1 \mathrm{~mm}^{2}, 90.7 \pm 21.2 \mathrm{~mm}^{2}$, and $54.4 \pm 30.6 \mathrm{~mm}^{2}$ in groups $\mathrm{A}, \mathrm{B}$, and C, respectively (Table 2, Fig. $2)$, and these areas were not significantly different $(P>0.05)$.

There was no evidence of unintentional injury to the abdominal organs, the abdominal wall, the skin, or the vertebral body on MRI in any animal.

\section{Histology}

Coagulation necrosis without structural damage was identified in all pancreas samples. No inflammatory cell infiltration was observed in or around the treated area in the pathologic specimens. An apparent boundary was noted between the normal and targeted tissues in all study groups (Fig. 3). Although fat necrosis was noted in the surrounding pancreatic parenchyma in all swine, architectural

Table 2. Area of the treated lesions on magnetic resonance imaging in each pig

\begin{tabular}{ccccc}
\hline Group & Pig & Dx $(\mathrm{mm})$ & Dy $(\mathrm{mm})$ & Area $\left(\mathrm{mm}^{2}\right)$ \\
\hline A & 1 & 12 & 6.2 & 58.4 \\
& 2 & 14.9 & 9.6 & 112.3 \\
B & 3 & N/A & N/A & N/A \\
& 4 & 12.2 & 7.9 & 75.7 \\
& 5 & 13.6 & 9.9 & 105.7 \\
C & 6 & 13.9 & 8.1 & 88.4 \\
& 7 & 7.7 & 4.8 & 29.0 \\
& 8 & 7.9 & 7.4 & 45.9 \\
\hline
\end{tabular}

Dx, transverse diameter of the lesion in the axial plane; Dy, vertical diameter of the lesion in the axial plane; N/A, no measurable area due to pseudocyst formation.

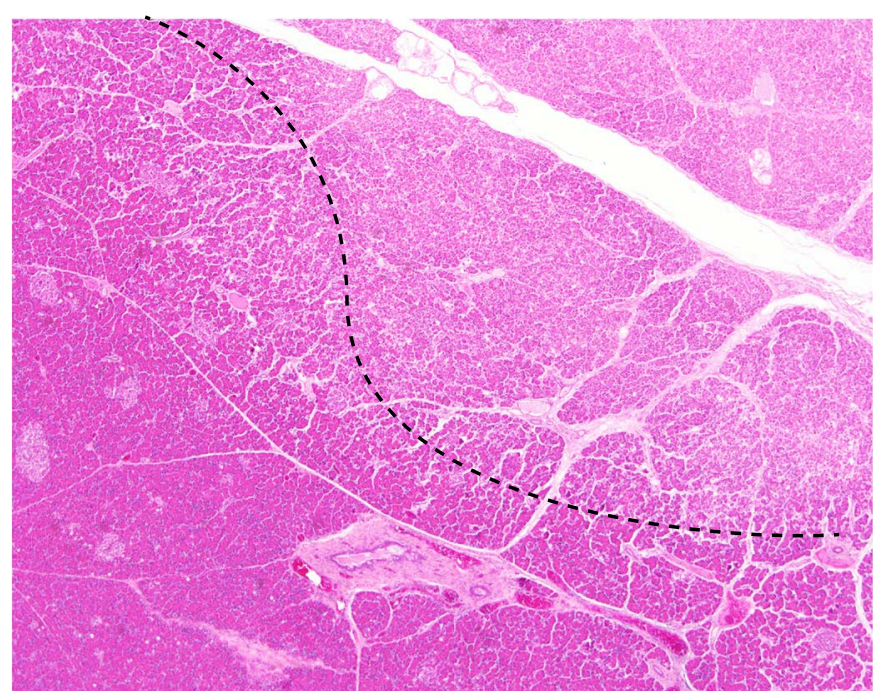

Fig. 3. Specimen of the pancreas with hematoxylin and eosin staining $(x 40)$. A clear demarcation between the normal and target tissues is seen (dotted line). 
distortions were not noted. In groups A and B, the treated areas showed homogeneous coagulation necrosis, but in two pigs in group $C$, patchy viable portions were noted in the ablation areas (Fig. 4). In the specimen from pig 1 (group A, using $1.7 \mathrm{~kW} / \mathrm{cm}^{2}$ ), coagulation necrosis was induced in the subcapsular area of the pancreas, but no significant adjacent organ injury was observed (Fig. 5).

\section{Discussion}

Our study showed that HIFU treatment of the pancreas using a new portable USg-HIFU machine was feasible and safe. Although previous studies have already demonstrated that USg-HIFU treatment for pancreatic cancer is feasible, none of the HIFU systems

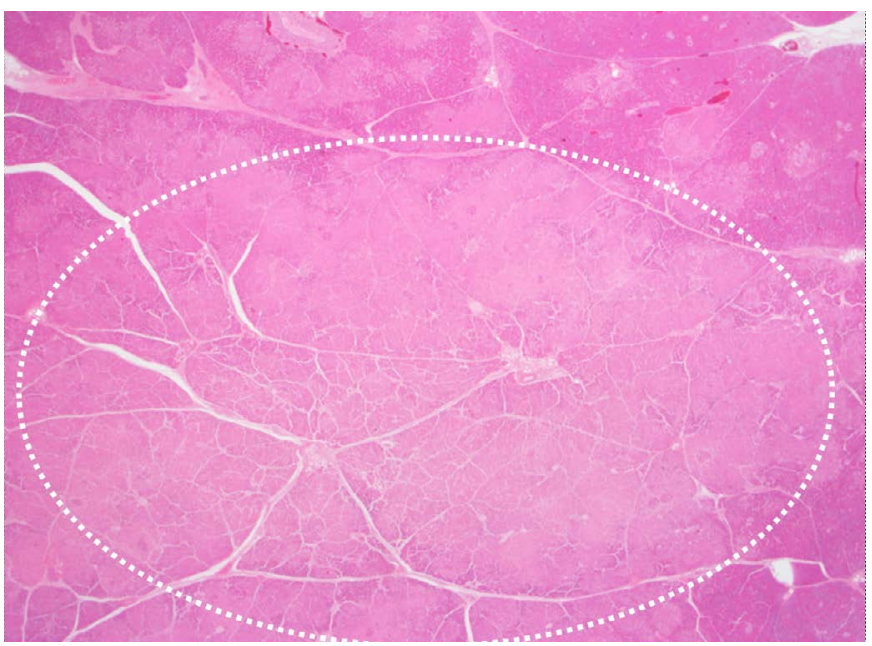

A

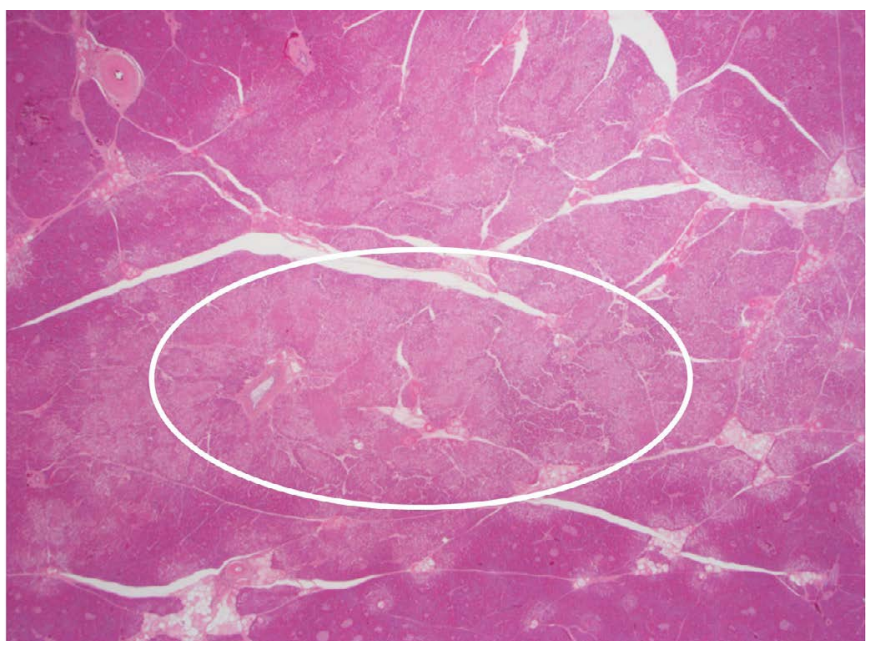

C

Fig. 4. Specimens of the pancreas with hematoxylin and eosin staining.

A, B. Specimens from groups A and B show homogeneous coagulation necrosis (circle) $(\times 12.5)$. C. A specimen from group $C$ shows patchy viable portions (circle) (x12.5). D. A specimen from group C shows patchy viable portions (arrows) $(\times 100)$.

e-ultrasonography.org

Ultrasonography 37(4), October 2018

303 


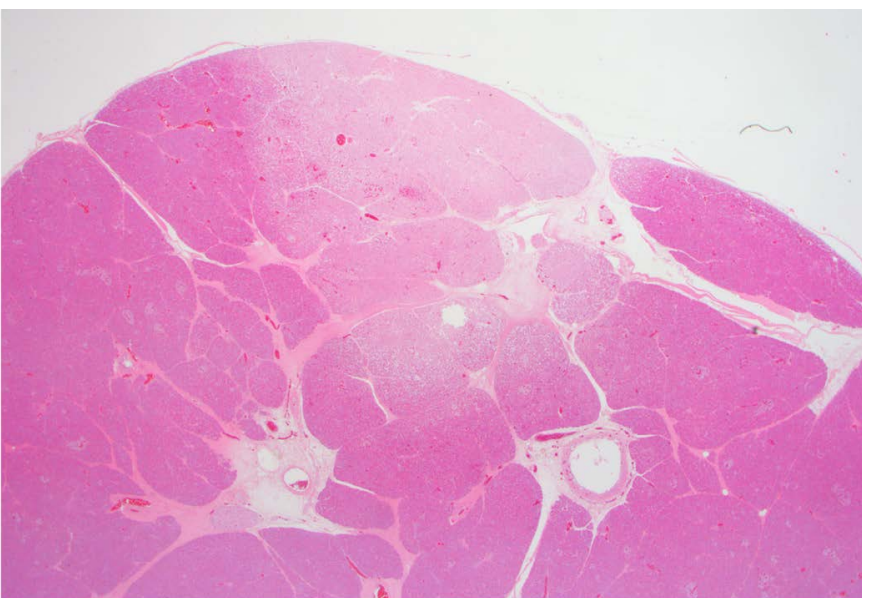

Fig. 5. Specimen of the pancreas in pig 1 (group A) (×12.5). A coagulation necrosis (pale pinkish area) is seen in the subcapsular region.

image display function and the electronic steering function, which enabled $2 \mathrm{D}$ or $3 \mathrm{D}$ ablation without moving the head unit, enhanced the accuracy of targeting and provided assurance to the operator.

Three different acoustic intensities were used in our study. To show the feasibility of ablating the pancreas, a relatively high intensity of $1.7 \mathrm{~kW} / \mathrm{cm}^{2}$ and a longer exposure duration of 8 seconds were used in the first pig (pig 1). Coagulation necrosis was achieved in the anterior subcapsular portion of the pancreas, but not exclusively within the pancreas. The parenchymal thickness was thinnest in the first pig, which could have contributed to this result. However, no significant adjacent organ injury was observed. Other possible causes may have included focusing HIFU anterior to the center of the pancreatic parenchyma, the greater acoustic intensity, and the longer ablation time. To generate an ablation lesion surrounded by normal pancreatic parenchyma, the treatment parameters were modulated. The delivered energy was reduced to an acoustic intensity of $1.5 \mathrm{~kW} / \mathrm{cm}^{2}$ and a shorter exposure duration of 7 seconds was used in the second pig (pig 2), and the targeting focus was placed slightly deeper than the center of the pancreatic parenchyma. Coagulation necrosis was then generated in the pancreas.

In a pig in group B, a pseudocyst developed in the pancreas. However, no abnormalities were found in the daily status observations, and no injuries were noted on gross examination or the MRI study. The levels of amylase and lipase did not significantly increase (29.9 U/L and $8 \mathrm{U} / \mathrm{L}$ on day 0 and $32.1 \mathrm{U} / \mathrm{L}$ and $4 \mathrm{U} / \mathrm{L}$ on day 7 , respectively). Therefore, despite the formation of a pseudocyst, this lesion was considered asymptomatic and safe enough to wait for spontaneous resolution. Hwang et al. [14] reported stomach and small bowel injuries as complications, and Xie et al. [15] reported skin burns and transient fatigue as minor complications. Although a direct comparison with the complications observed in other preclinical studies using other HIFU machines was difficult due to the different experimental settings, there were no significant complications in our study. Based on the results obtained for group $A$, an acoustic intensity of $1.5 \mathrm{~kW} / \mathrm{cm}^{2}$ was used in group $B$, and to check the effect of lower acoustic intensities, a lower acoustic intensity of $1.2 \mathrm{~kW} / \mathrm{cm}^{2}$ was applied in group C. There were no complications in any pigs. The area of coagulation necrosis was smaller in group $\mathrm{C}$, but this difference was not statistically significant. The specimens from two pigs in group $C$ showed patchy viable areas in the treated areas. In a previous in vivo study using an acoustic energy of $750 \mathrm{~J}$, a similar energy level to group $C$ in our study, an ablation area was not observed in some cases [14]. Therefore, some remaining viable area may have been present due to the use of inadequate energy to ablate the pancreatic tissue. In our study, HIFU treatment was performed without breath-holding or respiratory gating, so the targeted area could move during the treatment. Therefore, the energy delivered to the pancreatic parenchyma must be dispersed along the cranial and caudal directions. This may be a reason why viable tissue remained within the ablation territory. Although patchy viable areas were noted in the ablated lesions on pathologic specimens, the ablated areas on MRI were observed as hypovascular lesions, indistinguishable from the other ablated lesions showing homogeneous coagulation necrosis.

Recently, several studies have shown that the concurrent use of HIFU and chemotherapy increased the therapeutic effects of HIFU $[18,21,24]$. Kim et al. [25] reported that the concurrent use of gemcitabine and HIFU using $10 \%$ of the acoustic power used in routine HIFU for tissue ablation was more effective for tumor control than was the concurrent use of HIFU with high power. Yu et al. [26] found that the additional use of microbubbles enhanced the therapeutic effects of gemcitabine with low-acoustic-power HIFU. Therefore, a focused beam with a lower intensity than was used in this study can augment the effects of chemotherapy and may decrease the risk of complications.

Our study had a few limitations. First, a small number of animals and a narrow range of experimental conditions were used in our study. Therefore, differences or complications among the groups may have been obscured. Further studies with more animals and more diverse experimental conditions should be conducted. Second, we evaluated the safety of the procedure with a relatively short ablation time (79 or 88 seconds), which is much shorter than the usual treatment time in clinical studies. To ablate an entire tumor at an advanced stage, the treatment time may be increased to as long as several hours $[16,18,22]$, but the risk of complications may increase. Third, the tissue composition of normal pancreatic parenchyma is different from that of cancerous tissue. Thus, the ablation response 
may be different when HIFU is used to treat cancer [14]. Finally, although this HIFU device has the potential to enhance the accuracy of lesion targeting and to provide additional assurance to the operators due to the spatial flexibility of the treatment head, and to decrease the total procedure time through 3D electronic steering, we did not compare those factors with other devices. Further studies should be performed to evaluate those potential benefits.

In conclusion, pancreatic ablation using a new, portable USgHIFU system was safe and feasible in a swine model. Coagulation necrosis of the pancreas was successfully achieved with various acoustic intensities.

ORCID: Won Chang: http://orcid.org/0000-0001-7367-9841; Jae Young Lee: http:// orcid.org/0000-0001-6946-6042; Jae Hwan Lee: http://orcid.org/0000-0001-65133224; Jae Seok Bae: http://orcid.org/0000-0003-2768-7917; Keonho Son: http:// orcid.org/0000-0002-0749-730X; Joon Koo Han: http://orcid.org/0000-0001-59165545

\section{Conflict of Interest}

No potential conflict of interest relevant to this article was reported.

\section{Acknowledgments}

This work was supported in part by the R\&D program of MOTIE/KEIT (10043474, Development of an Ultrasound Guided HIFU Treatment System) and in part by a grant from the Korea Health Technology R\&D Project through the Korea Health Industry Development Institute (KHIDI), which was funded by the Ministry of Health \& Welfare, Republic of Korea (grant number: HI15C1532).

\section{References}

1. Stewart BW, Wild CP. World cancer report 2014. Geneva: World Health Organization, International Agency for Research on Cancer, 2014.

2. Konstantinidis IT, Warshaw AL, Allen JN, Blaszkowsky LS, Castillo CF, Deshpande V, et al. Pancreatic ductal adenocarcinoma: is there a survival difference for $\mathrm{R} 1$ resections versus locally advanced unresectable tumors? What is a "true" R0 resection? Ann Surg 2013:257:731-736.

3. Hariharan $D$, Saied A, Kocher HM. Analysis of mortality rates for pancreatic cancer across the world. HPB (Oxford) 2008;10:58-62.

4. von Wichert G, Seufferlein T, Adler G. Palliative treatment of pancreatic cancer. J Dig Dis 2008;9:1-7.

5. Siegel RL, Miller KD, Jemal A. Cancer statistics, 2016. CA Cancer J Clin 2016;66:7-30.

6. Orsi F, Arnone P, Chen W, Zhang L. High intensity focused ultrasound ablation: a new therapeutic option for solid tumors. J
Cancer Res Ther 2010;6:414-420.

7. Zhou Y. High-intensity focused ultrasound treatment for advanced pancreatic cancer. Gastroenterol Res Pract 2014;2014:205325.

8. Farny $\mathrm{CH}$, Holt RG, Roy RA. Temporal and spatial detection of HIFU-induced inertial and hot-vapor cavitation with a diagnostic ultrasound system. Ultrasound Med Biol 2009;35:603-615.

9. Hsieh CY, Probert Smith P, Mayia F, Ye G. An adaptive spectral estimation technique to detect cavitation in HIFU with high spatial resolution. Ultrasound Med Biol 2011;37:1134-1150.

10. Nandlall SD, Jackson E, Coussios CC. Real-time passive acoustic monitoring of HIFU-induced tissue damage. Ultrasound Med Biol 2011:37:922-934

11. Henderson PW, Lewis GK, Shaikh N, Sohn A, Weinstein AL, Olbricht WL, et al. A portable high-intensity focused ultrasound device for noninvasive venous ablation. J Vasc Surg 2010;51:707-711.

12. Tobias RJ, Uvacek B, Wu BW. A portable dual-mode ultrasound platform with multi-rail voltage power supply for adaptive diagnostic imaging and therapy sequence programming. In: 2015 IEEE International Ultrasonics Symposium (IUS); 2015 Oct 21-24; Taipei, Taiwan; INSPEC accession No. 15601759.

13. Choi JW, Lee JY, Hwang EJ, Hwang I, Woo S, Lee CJ, et al Portable high-intensity focused ultrasound system with 3D electronic steering, real-time cavitation monitoring, and 3D image reconstruction algorithms: a preclinical study in pigs. Ultrasonography 2014;33:191-199.

14. Hwang JH, Wang YN, Warren C, Upton MP, Starr F, Zhou Y, et al. Preclinical in vivo evaluation of an extracorporeal HIFU device for ablation of pancreatic tumors. Ultrasound Med Biol 2009;35:967975.

15. Xie B, Li YY, Jia L, Nie YQ, Du H, Jiang SM. Experimental ablation of the pancreas with high intensity focused ultrasound (HIFU) in a porcine model. Int J Med Sci 2010;8:9-15.

16. Wu F, Wang ZB, Zhu H, Chen WZ, Zou JZ, Bai J, et al. Feasibility of US-guided high-intensity focused ultrasound treatment in patients with advanced pancreatic cancer: initial experience. Radiology 2005:236:1034-1040.

17. Xiong LL, Hwang JH, Huang XB, Yao SS, He CJ, Ge XH, et al. Early clinical experience using high intensity focused ultrasound for palliation of inoperable pancreatic cancer. JOP 2009;10:123-129.

18. Lee JY, Choi BI, Ryu JK, Kim YT, Hwang JH, Kim SH, et al. Concurrent chemotherapy and pulsed high-intensity focused ultrasound therapy for the treatment of unresectable pancreatic cancer: initial experiences. Korean J Radiol 2011;12:176-186.

19. Kovatcheva R, Guglielmina JN, Abehsera M, Boulanger L, Laurent $\mathrm{N}$, Poncelet E. Ultrasound-guided high-intensity focused ultrasound treatment of breast fibroadenoma-a multicenter experience. J Ther Ultrasound 2015;3:1.

20. Kovatcheva RD, Vlahov JD, Stoinov JI, Zaletel K. Benign solid thyroid nodules: US-guided high-intensity focused ultrasound ablation- 
initial clinical outcomes. Radiology 2015;276:597-605.

21. Zhao $H$, Yang G, Wang D, Yu X, Zhang Y, Zhu J, et al. Concurrent gemcitabine and high-intensity focused ultrasound therapy in patients with locally advanced pancreatic cancer. Anticancer Drugs 2010;21:447-452.

22. Jung SE, Cho SH, Jang JH, Han JY. High-intensity focused ultrasound ablation in hepatic and pancreatic cancer: complications. Abdom Imaging 2011;36:185-195.

23. Li PZ, Zhu SH, He W, Zhu LY, Liu SP, Liu Y, et al. High-intensity focused ultrasound treatment for patients with unresectable pancreatic cancer. Hepatobiliary Pancreat Dis Int 2012;11:655-660.

24. Wang RS, Liu LX, Gu YH, Lin QF, Guo RH, Shu YQ. The effect of endostatin and gemcitabine combined with HIFU on the animal xenograft model of human pancreatic cancer. Biomed Pharmacother 2010;64:309-312.

25. Kim JH, Kim H, Kim YJ, Lee JY, Han JK, Choi BI. Dynamic contrastenhanced ultrasonographic (DCE-US) assessment of the early response after combined gemcitabine and HIFU with low-power treatment for the mouse xenograft model of human pancreatic cancer. Eur Radiol 2014;24:2059-2068.

26. Yu MH, Lee JY, Kim HR, Kim BR, Park EJ, Kim HS, et al. Therapeutic effects of microbubbles added to combined high-intensity focused ultrasound and chemotherapy in a pancreatic cancer xenograft model. Korean J Radiol 2016;17:779-788. 